\title{
Integrative Oncology_Finding an Indian Solution to Universal Problems for Holistic Cancer Care!!
}

\author{
Chintamani $^{1}$
}

Published online: 6 August 2015

(C) Indian Association of Surgical Oncology 2015

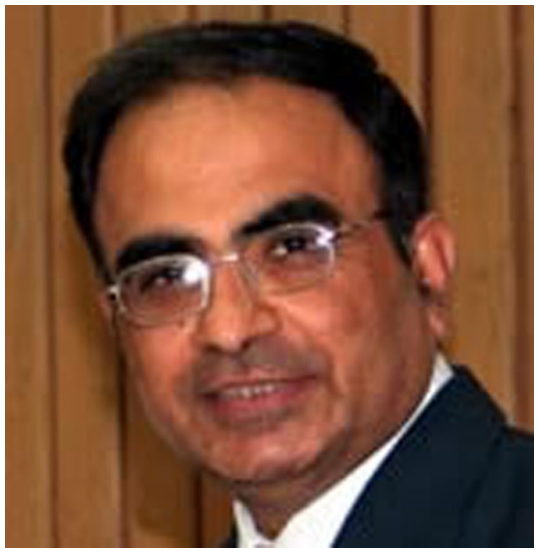

Integrative oncology (IO) involves combining the best of modern medicine with the "evidence based" natural, complementary or traditional medicine. IO is gaining great importance with the management of cancer patients becoming more and more holistic. It is essential to take care of the bio-psycho-social and spiritual needs of these patients before, during and after treatment and various complimentary therapies are being employed to address this issue. This holistic approach is required at all levels of treatment to not only potentiate the role of established modalities but also to deal with toxicities related to them.

It is important to answer one very vital question before we incorporate these therapies in to our routine protocols, "is cancer

\section{Chintamani}

drchintamani7@gmail.com

1 Vardhman Mahavir Medical College, Safdarjang Hospital, New Delhi, India therapy negotiable or can we side step it"? The answer to this question is a certain "No" and we need evidence-based therapies even for the so-called "grey zones" or "third space" in oncology. Integrated oncology (IO) is about using modalities like Yoga and indigenous herbs to not only attempt treating cancer but also improving the quality of life. Most cancer patients suffer from anxiety and depression that is related to disease and its therapies. This IO concept offers revolutionary possibilities for a patient struggling to survive, by contributing to the quality of life [1-4].

Instead of the patient getting held up in a gloomy dark and scary world of cancer once the news is broken, these therapies offer a let off and hope for a positive outcome. This in turn also empowers these patients to develop strength to meet and live with illness. It is well known that often "cancer treatment can become worse than the disease" and we need to address the "whole" patient rather than just the disease. The dropout rates during cancer therapy are well known, especially in the developing countries and these therapies can keep these patients motivated, engaged and improve compliance. It is vital to engage and encourage cancer patients so that they don't run away from the disease and should instead be motivated in to living with it joyfully [1-6].

\section{Yoga and Healing}

The rich tradition of Yoga has very deep roots in India and was started by sage Patanjali [1-3]. It has got refined over centuries as an established scientific "way" of life. This technique has a unique power to alleviate pain, sorrow and stimulate healing by encouraging the learner in to working with his/ her senses and intellect. By improving the immune system and stimulating release of endorphins, yoga has been shown to not only help improve feeling of well being but also better response to therapies in some studies [1,2]. 


\section{The Way Yoga Works}

Initially the cancer patient is benefitted by asanas that help in exercising every muscle, nerve and gland in the body. These postures can be tailored to the patient and his disease. The suttle movements help in addressing the tension that is holding and also blocking energy in any organ or system. With the release of tension the energy can flow more freely leading to a sense of well being and increasing the strength of body, mind and spirit. The growth of tumors and the other indicators have been found to increase with chronic cancer induced stress that takes away the function of "killer cells" that are responsible for our defences. This makes us more vulnerable to the growth and spread of tumours [2-7].

\section{Yoga Therapy in Integrative Oncology}

Yoga is now widely accepted and is available in most recognized centres around the world. This therapy is being used along with touch-based therapies like reiki, reflexology, counselling, art therapy and meditation that can be offered individually or in small groups. Being evidence based and a very gentle exercise and meditation, yoga has been easily accepted and incorporated as a useful treatment modality for cancer. However claims that yoga can "cure" cancer are without any scientific evidence and should not be accepted. But as a complementary modality the role of Yoga has been established [2, 3].

There is also a flip side to the entire story and natural and complementary medicine world is rife with conspiracy theories. Often-tall claims are made that can confuse patients and we need to put issues in perspective. It is therefore mandatory to have a strong regulatory body to take serious look at the curriculum of various courses run in the country to ensure evidence based, accountable and reproducible therapies. More controlled research is also needed to increase the database and reach some authentic conclusions that can be used for the good of cancer patients.

Yoga has been studied extensively and there is now a large data of evidence to support its use in oncology. The benefits of yoga therapy are largely psychological but there are many reports to suggest physiological benefits to support its use in cancer patients. There is decrease in fear of recurrence and insomnia observed in various studies [2, 4-7].

\section{Mind Fullness Based Stress Reduction [MBSR]}

The basis to various beneficial effects of yoga have been attributed to increased mindfulness and shift from sympathetic nervous system to parasympathetic nervous system known as mind fullness based stress reduction. This has been found to be very useful in the pains induced by aromatase inhibitors; post surgery or cancer related or induced pains [2, 3, 6, 7].

"Chemo brain" is a cognitive impairment induced by chemotherapy and yoga has been found to be beneficial in this syndrome especially in the elderly. Lymphedema, a usual morbidity after lymph node dissections for cancer has been shown to decrease with yoga with improvement of upper body range of motions. Nausea, a regular toxicity with chemotherapy for breast cancer has been found to be reduced by yoga therapy. There is also a significant improvement in digestion following Yoga in these patients. Canadian Yoga Thrive programme found great improvement in quality of life and digestive functions and cancer/chemotherapy related digestive stress [2, 3, 5-7].

Integrative oncology is thus fast becoming an integral part of cancer care and yoga forms the most important part of it. There is now ample evidence to support that yoga takes care of mood disturbance and distress in cancer patients, which goes a long way in to improving compliance and also response to therapy. A lot has been done and is being done in the field but we need to do more to find "Indian solutions" for the rest of the world, to address the "grey zone" or the "third space" of oncology and yoga can be an important tool in our hands.

\section{References}

1. Chintamani, Gogne A, Khandelwal R, Tandon M, Jain S, Kumar Y, Narayan N, Bamal R, Srinivas S, Saxena S (2011) The correlation of anxiety and depression levels with response to neoadjuvant chemotherapy in patients with breast cancer. JRSM Short Rep 2(3):15

2. Milano C (2002) How yoga is being incorporated into cancer center offerings. Oncol Times 24(3):79-80

3. Banerjee B, Vadiraj HS, Ram A, Rao R, Jayapal M, Gopinath KS, Ramesh BS, Rao N, Kumar A, Raghuram N, Hegde S, Nagendra HR, Prakash Hande M (2007) Effects of an integrated yoga program in modulating psychological stress and radiation-induced genotoxic stress in breast cancer patients undergoing radiotherapy. Integr Cancer Ther 6(3):242-250

4. Carson J et al (2007) Yoga for women with metastatic breast cancer: results from a pilot study. J Pain Symptom Manag 33(3):331-341

5. Carson J et al (2009) Yoga of awareness program for menopausal symptoms in breast cancer survivors: results from a randomized trial. Support Care Cancer. doi:10.1007/s00520-009-0587-5

6. Chandwani Kavita D, Thornton B, Perkins GH, Arun B, Raghuram NV, Nagendra HR, Wei Q, Cohen L (2010) Yoga improves quality of life and benefit finding in women undergoing radiotherapy for breast cancer. J Soc Integr Oncol 8(2):43-55

7. Culos-Reed SN, Carlson LE, Daroux LM, Hately-Aldous S (2006) A pilot study of yoga for breast cancer survivors: physical and psychological benefits. Psychooncology 15(10):891-897 